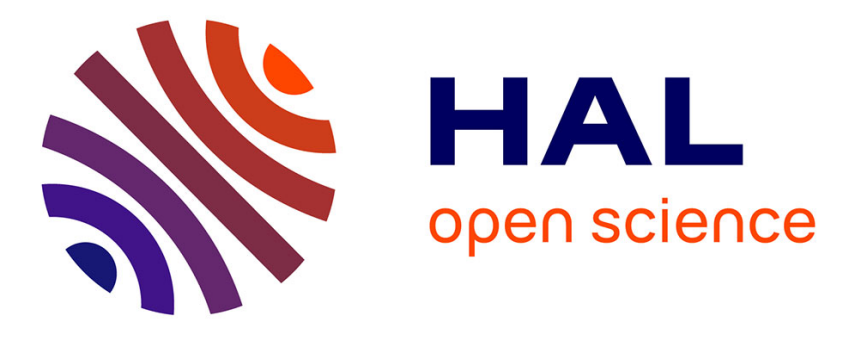

\title{
Modeling patient-specific desaturation patterns in sleep apnea
}

\author{
Gustavo Guerrero, Virginie Le Rolle, Corinne Loiodice, Amel Amblard, \\ Jean-Louis Pepin, Alfredo I Hernandez
}

\section{To cite this version:}

Gustavo Guerrero, Virginie Le Rolle, Corinne Loiodice, Amel Amblard, Jean-Louis Pepin, et al.. Modeling patient-specific desaturation patterns in sleep apnea. IEEE Transactions on Biomedical Engineering, 2022, 69 (4), pp.1502-1511. 10.1109/TBME.2021.3121170 . hal-03477355

\section{HAL Id: hal-03477355 https://hal.science/hal-03477355}

Submitted on 14 Dec 2021

HAL is a multi-disciplinary open access archive for the deposit and dissemination of scientific research documents, whether they are published or not. The documents may come from teaching and research institutions in France or abroad, or from public or private research centers.
L'archive ouverte pluridisciplinaire HAL, est destinée au dépôt et à la diffusion de documents scientifiques de niveau recherche, publiés ou non, émanant des établissements d'enseignement et de recherche français ou étrangers, des laboratoires publics ou privés.

\section{다)(1) $(5$}

Distributed under a Creative Commons Attribution - NonCommercial| 4.0 International 


\title{
Modeling patient-specific desaturation patterns in sleep apnea
}

\author{
Gustavo Guerrero, Virginie Le Rolle, Corinne Loiodice, Amel Amblard, \\ Jean-Louis Pépin, and Alfredo Hernández,Member, IEEE .(e-mail: virginie.lerolle@univ-rennes1.fr).
}

\begin{abstract}
Objective: The physiological mechanisms involved in cardio-respiratory responses to sleep apnea events are not yet fully elucidated. A model-based approach is proposed to analyse the acute desaturation response to obstructive apneas. Methods: An integrated model of cardio-respiratory interactions was proposed and parameters were identified, using an evolutionary algorithm, on a database composed of 107 obstructive apneas acquired from 10 patients (HYPNOS clinical study). Unsupervised clustering was applied to the identified parameters in order to characterize the phenotype of each response to obstructive apneas. Results: A close match was observed between simulated oxygen saturation $\left(\mathrm{SaO}_{2}\right)$ and experimental $\mathrm{SaO}_{2}$ in all identifications (median RMSE = 1.3892 $\%)$. Two clusters of parameters, associated with different dynamics related to sleep apnea and periodic breathing were obtained. Conclusion and significance: The proposed patient and event-specific model-based analysis provides understanding on specific desaturation patterns, consequent to apnea events, with potential applications for personalized diagnosis and treatment.
\end{abstract}

Index Terms - Sleep Apnea, Phenotyping, Mathematical model, Patient-specific identification

\section{INTRODUCTION}

$\mathbf{S}$ LEEP apnea syndrome (SAS) is a multifactorial condition characterized by repeated episodes of cessation of breathing (apnea) or important reductions in breathing amplitude (hypopnea) during sleep. Patients with SAS can suffer from 5 to up to 100 apnea or hypopnea episodes per hour of sleep, with duration going from 10 seconds to several minutes, producing acute cardio-respiratory responses and sleep fragmentation [1]. In the long term, these acute effects increase the risk of suffering various chronic conditions such as hypertension, stroke, heart disease and metabolic disorders [1].

SAS is affecting nearly one billion people worldwide [2]. The gold-standard for the diagnosis of SAS is polysomnography (PSG), which consists of a complete multi-channel recording and monitoring of cardio-respiratory and sleep signals

Paper submitted on March 2021. Results incorporated in this publication received funding from the European Union's Horizon 2020 research and innovation program under grant agreement $\mathrm{N}^{\circ} 689260$ (Digi-NewB project) and the ANR TecSan PASITHEA project.

G. Guerrero, V. Le Rolle and A. Hernndez are with Univ Rennes, Inserm, LTSI - UMR 1099, F-35000 Rennes, France

A. Amblard is with Sorin CRM SAS - Microport, Clamart, France.

C. Loiodice and J. Ppin are with Grenoble Alpes University, HP2 laboratory, Inserm, U1042, Grenoble, F-38000, France and with Grenoble Alps University Hospital, Department of Physiology and Sleep, Grenoble, F-38000,France. during a whole night. The interpretation of acute responses due to apneas and hypopneas can be difficult because of the variety of processes involved (autonomic regulation, ventilation, chemoreflexes, etc.), which should be jointly considered for an appropriate analysis. As a consequence, new methods are needed to provide a better understanding of physiological mechanisms involved in the cardio-respiratory responses to apneas. In this context, a model-based approach seems particularly adapted because it allows for the explicit integration of physiological knowledge into the data processing task and for an explainable analysis of the underlying mechanisms.

Most of the existing computational models involving cardiovascular or respiratory functions represent isolated aspects of physiology, or do not take into account the main physiological mechanisms underlying apnea [3]-[5]. Only a few integrated models of cardio-respiratory interactions have been proposed [6]-[8] integrating the most important mechanisms to analyse sleep apneas. The models proposed in [6] and [7] are capable of simulating obstructive apneas and periodic breathing, but they are highly parameterized representations, focused on comparing the model responses with the empirically derived responses that represent the average subject in each patient/subject population. The model proposed in [8] can simulate the dynamics related to hypercapnia and hypoxia, but the difficulties for defining patient-specific parameters for this model has been presented as a limitation. None of these models have been used in patient-specific applications, due to their complexity.

Our team has proposed a number of integrated models including parameter identification from clinical data in the cardiovascular field [9], [10], in neuromodulation applications [11] and for the analysis of the respiratory system [12], [13].

This paper presents a model-based analysis of the cardiorespiratory interactions during obstructive sleep apnea events, using an original cardio-respiratory model. The objectives of this work are: $i$ ) to propose a patient-specific and event-specific modeling for the desaturation response to apnea events, ii) to analyze the dynamics of $\mathrm{SaO}_{2}$ during an obstructive event in a patient-specific manner, iii) to characterize the response to apneic events, clustering the results for identification into "phenotypes" of responses. A preliminary version of this work has been reported in [14].

\section{MethODS}




\section{A. Integrated cardio-respiratory model}

The proposed model (Figure 1) is composed of four interconnected components : i) the respiratory system, ii) the cardiovascular system, iii) the gas exchange (lungs and metabolism) and iv) the neural control. The combined model is characterized by 42 state variables and 151 parameters and it was implemented using the Multiformalism Modeling and Simulation Library (M2SL) [15]. Equations and parameters of this model can be found in the supplementary online material.

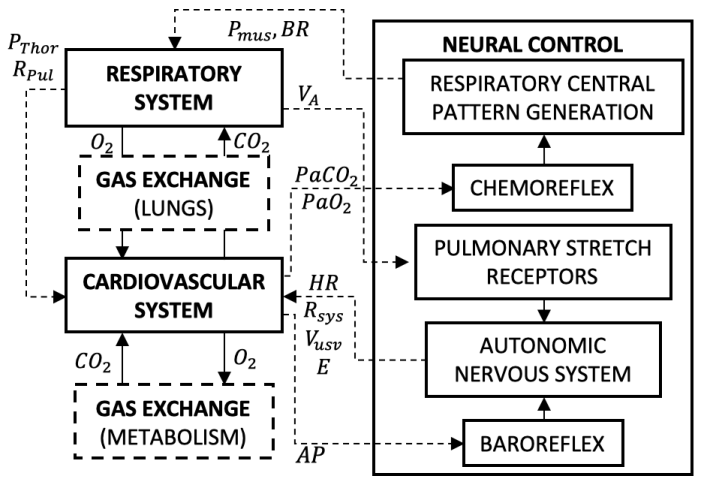

Fig. 1. Diagram of the cardio-respiratory adult model. Dotted line arrows symbolize interactions between sub-models. $\boldsymbol{P}_{\text {thor }}$, thoracic pressure; $\boldsymbol{R}_{\text {pul }}$ pulmonary capillaries resistance; $\boldsymbol{P} \boldsymbol{m u s}$, respiratory muscles pressure; $\mathrm{BR}$, breathing rate; $\boldsymbol{V}_{\boldsymbol{A}}$, alveolar volume; $\mathrm{PaO}_{2}$ and $\mathrm{PaCO}_{2}$, partial pressure of $\mathrm{O}_{2}$ and $\mathrm{CO}_{2}$ in the systemic arteries, respectively; HR, heart rate; Rsys, systemic peripheral resistance; $\boldsymbol{V u s v}$, unstressed volume of the systemic veins; $\boldsymbol{E}$, ventricle elastances; $\boldsymbol{A P}$ arterial pressure.

1) Cardiovascular system model: The cardiovascular system (CVS) presented in Figure 2 is an adaptation of the previous models of our group [10], [11]. It is constituted of three coupled components: (a) the cardiac electrical system, (b) cardiac cavities and (c) systemic and pulmonary circulation.

a) Cardiac electrical system: The cardiac electrical conduction system (CES model) is based on a simplified discrete model adapted from [16]. It is defined as a set of interconnected generalized automata, each one representing the electrical activation of a group of cardiac cells: the sinoatrial node, the left atrium (LA), the atrioventricular node, the upper bundle of His, the lower bundle of His, left and right bundle branches, and left and right ventricles (LV and RV). The output electrical activations of LV and LA are connected to the CVS model by triggering the mechanical contractions of the ventricles and the atrial chambers respectively.

b) Cardiac cavities: The mechanical function of the left and right heart (ventricles and atria) and the cardiac valves are represented in the CVS model. The cardiac cavity pressure is represented by a combination of end-systolic and enddiastolic pressure-volume relationships in each compartment. Each cardiac cavity is represented by a time-variant elastance, controlled by a driving function that represents contraction and relaxation phases. The elastance of the atrium is defined by a Gaussian function [17] while the elastance of the ventricles is defined by a Two-hill driving function triggered by the CES system [18].
Volume $(V)$ in each cavity is calculated from the integral of their respective net flow. The cardiac valves are represented as diodes.

c) Cardiovascular system circulation: The cardiovascular circulation is divided into the pulmonary and systemic circulations, also including the difference between intrathoracic and extrathoracic compartments. Pressures $(P)$ in each compartment are calculated as a linear relationship between its volume $(V)$ and vascular elastance $(E)$. Intrathoracic compartments are affected by the thoracic pressure $\left(P_{\text {thor }}\right)$ of the respiratory sub-model. These pressures are then used to calculate flows between chambers as: $Q=\frac{\Delta P}{R}$ where $\Delta P$ is the pressure gradient of two chambers and $R$ is the corresponding vascular resistance connecting them.

In order to integrate the metabolic gas exchange sub-model, a new compartment representing the systemic peripheral vessels has been included into the systemic circulation, following the structure of [8]. A pulmonary shunt compartment parallel to the pulmonary peripheral vessels was included in the pulmonary circulation. Resistance $\left(R_{p s} ; R_{p p}\right)$ and compliance values $\left(C_{p s}, C_{p p}\right)$ of these two compartments have been assigned to distribute the desired percentage of blood flow $(\mathrm{fs})$ coming out of the pulmonary arteries to the pulmonary shunt, as presented in eq. 1 and 2 .

$$
\begin{aligned}
& R_{p s}=R_{p p} \cdot \frac{(1.0-f s)}{f s} \\
& C_{p s}=C_{p p} \cdot \frac{f s}{(1.0-f s)}
\end{aligned}
$$

Moreover, the effect of lung air volume in lung perfusion was also integrated through the following equation [19]:

$$
R_{p a}\left(V_{A}\right)=R_{p a, 0} \cdot{\frac{V_{A}}{V_{A, \max }}}^{2}
$$

where $R_{p a}$ is the resistance of pulmonary arteries, $R_{p a, 0}$ is a constant to set the normal resistance value of the pulmonary arteries, $V_{A}$ is the alveolar volume and $V_{A, \max }$ is the maximum alveolar volume.

2) Respiratory model: The respiratory model (Figure 3 ) was adapted from previous work of our team [5] and previous models in the literature [20]. It includes the upper airways, the intermediate airways, the lower airways, the alveolar compartment, the pleural cavity, the chest wall and the respiratory muscles. The tracheobronchial airways are characterized by nonlinear relationships, while the alveolar space, the intermediate airway compartment and the chest wall were assumed to have a constant, purely elastic behavior and were represented by the constant compliances $C_{A}, C_{c}$ and $C_{c w}$ respectively. In order to represent realistic respiratory pressure and flow profiles, a respiratory muscles pressure function $P_{m u s}$ defined by [5] was used:

$$
P_{\text {mus }}=P_{\text {max }} \cdot\left(B_{o}+\left(1-B_{o}\right) \cdot\left(\frac{t_{r}}{T_{I}}\right)^{\beta} \cdot e^{1-\left(\frac{t_{r}}{T_{I}}\right)^{\alpha}}\right)
$$

where $P_{\max }$ corresponds to maximum muscle activity, $B_{0}$ is the basal level at end-expiration, $t_{r}$ is the time elapsed from the onset of the current respiratory cycle and $T_{I}$ is 


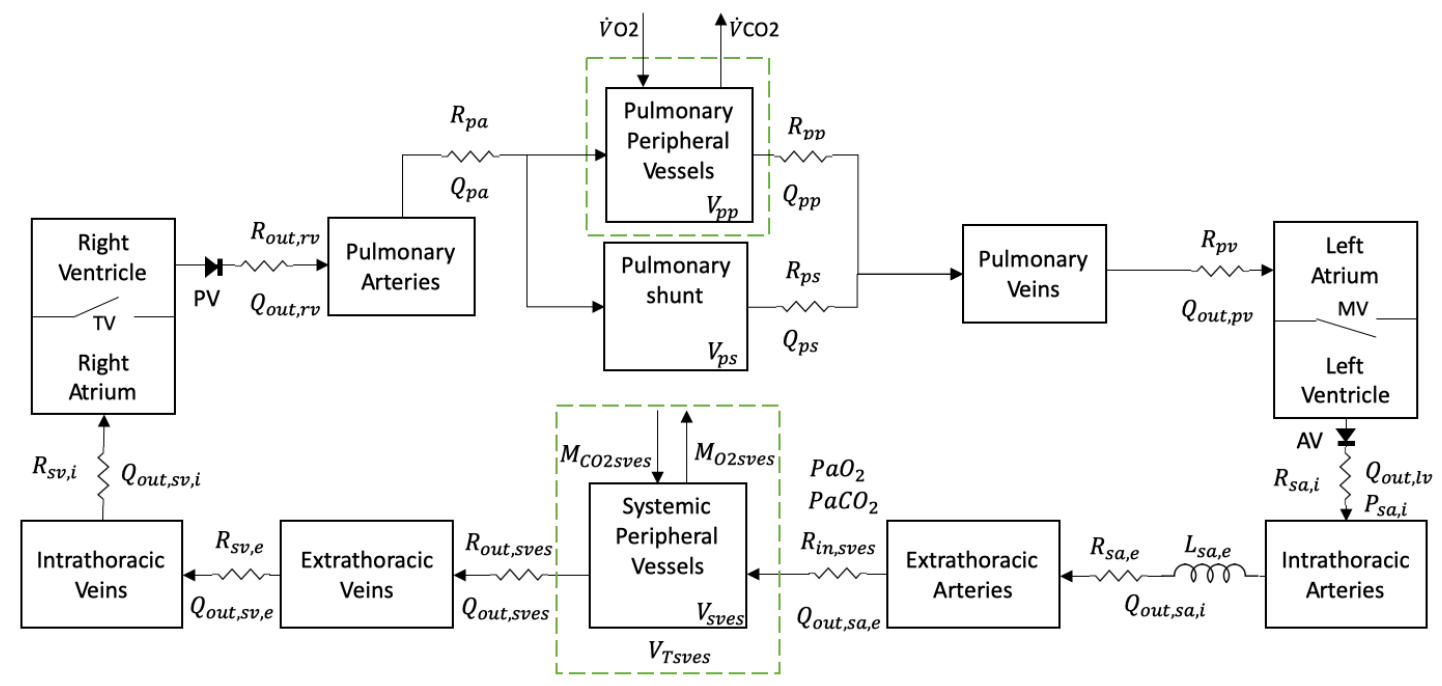

Fig. 2. Diagram of the cardiovascular system model. Each compartment is characterized by an elastance $(\boldsymbol{E})$ and its pressure $(\boldsymbol{P})$ and volume $(\boldsymbol{V})$ are computed. Green dotted line blocks correspond to the interface with the gas exchange sub-models. $\boldsymbol{Q}$, flow; $\boldsymbol{R}$, resistance; $\boldsymbol{L}$, inertia; ra, right atrium; rv, right ventricle; la, left atrium; Iv, left ventricle; tv, tricuspid valve; mv, mitral valve; pa, pulmonary arteries; pp, pulmonary peripheral; pv, pulmonary veins; sa, systemic arteries ;sves, systemic peripheral vessels; sv, systemic veins; i, intra-thoracic; e, extra-thoracic; $M$, metabolic rates; $\dot{\boldsymbol{V}}$, flow. The dotted-line compartments represent the gas exchange: Top: Lung gas exchange in the pulmonary peripheral vessels; Bottom: Metabolic gas exchange in the systemic peripheral vessels.

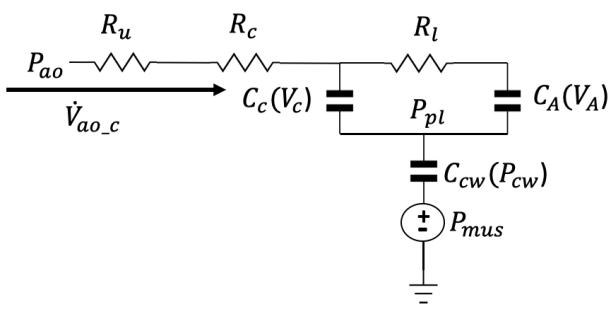

Fig. 3. Electric diagram of the respiratory system model. $\boldsymbol{P}$, pressure; $\boldsymbol{R}$ resistance; $\boldsymbol{V}$, volume; $\boldsymbol{C}$, compliance; $\dot{\boldsymbol{V}}$, airflow; ao, airway opening; u, upper airway; c, intermediate airways; I, lower airways; pl, pleural; cw, chest wall; A, alveolar; mus, respiratory muscles. $\dot{V}_{a o_{-}}$, airflow from the airway opening to the lower airways, also denominated lung respiratory flow.

the inspiration duration. In addition, parameters $\alpha$ and $\beta$ characterize the $P_{m u s}$ signal profile during inspiration and expiration, respectively. Thoracic pressure $\left(P_{\text {thor }}\right)$ is assumed to be equal to pleural pressure $\left(P_{p l}\right)$. The parameter $P_{a o}$ (airway opening pressure) can be used to simulate the effects of CPAP [13].

3) Gas exchange model: This sub-model is composed of three components: a) lung gas exchange, b) metabolism gas exchange and c) gas transport.

a) Lung gas exchange: The lung gas exchange model describes the exchanges of $\mathrm{CO}_{2}$ and $\mathrm{O}_{2}$ between the dead space compartment, the alveoli compartment and the pulmonary capillaries, as presented in Figure 4. This was adapted from [4], [8].

The model has several inputs from other sub-models:

1) The intermediate airway volume $\left(V_{c}\right)$ as the volume of the dead space, the alveolar volume $\left(V_{A}\right)$ and flow $\dot{V}_{A}$, and the lung respiratory flow $\dot{V}_{l}$ from the respiratory model.

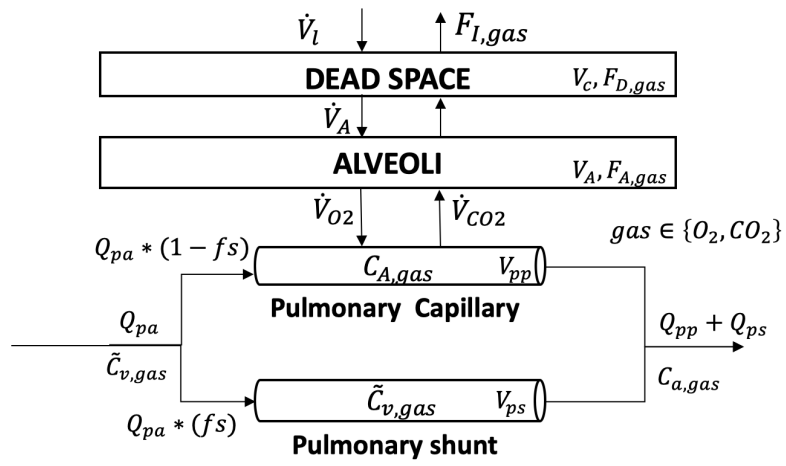

Fig. 4. Lung gas exchange model. $\boldsymbol{F}$, gas fraction; $\boldsymbol{V}$, volume; $\boldsymbol{Q}$, blood flow; $C$, concentration; I, inspired; D, dead space; A, alveolar; a, arterial; v, venous; out,pa, pulmonary capillaries; fs, shunt fraction; I, lung.

2) The pulmonary peripheral volume $\left(V_{p p}\right)$ and the blood flow through the pulmonary artery, peripheral circulation, and shunt $\left(Q_{p a}, Q_{p p}\right.$, and $\left.Q_{p s}\right)$ from the CVS model.

3) The delayed venous gas concentrations in the blood $\left(\tilde{C}_{v, \mathrm{O} 2}, \tilde{C}_{v, \mathrm{CO} 2}\right)$ from the metabolic gas exchange and gas transport model.

The fractions of inspired air $\left(\mathrm{FIO}_{2}, \mathrm{FICO}_{2}\right)$ are an external input to the model. Fractions and partial pressures of each gas are computed applying conservation of mass to each of the three compartments for each gas, assuming that every compartment is homogeneous and perfectly mixed. The relation between fractions and partial pressure of a gas is determined using the ideal gas law. The conversion from gas concentrations to partial pressures and vice-versa are calculated using the oxygen dissociation functions proposed in [21]. Equilibrium between pulmonary capillaries and alveoli in terms of 
gas partial pressures is assumed to happen instantaneously. The outputs of the model are the concentrations of gases in the pulmonary capillaries $\left(\mathrm{C}_{\text {A, gas }}\right.$, gas $\left.\in\left\{\mathrm{O}_{2}, \mathrm{CO}_{2}\right\}\right)$. These concentrations are converted to arterial blood gas concentrations $\left(C_{a, g a s}\right)$ mixing the pulmonary capillary and shunted blood. The pulmonary shunt represents the blood that does not take part in the lung gas exchange, hence it keeps the venous gas concentrations $\left(\tilde{C}_{v, \text { gas }}\right)$.

b) Metabolic gas exchange: A model of $\mathrm{CO}_{2}$ production and $\mathrm{O}_{2}$ consumption by the tissues and organs was integrated in the systemic peripheral vessels compartment of the CVS (Figure 2) as defined by [8]. This blood-tissue compartment is modelled as a simple container with a total volume given by the sum of the constant tissue volume $\left(V_{T, \text { sves }}\right)$ and the blood volume of the systemic peripheral vessels $V_{\text {sves }}$. Metabolic $\mathrm{O}_{2}$ consumption and $\mathrm{CO}_{2}$ production are assumed to happen at constant rates ( $M_{O 2 \text { sves }}$ and $M_{C O 2 s v e s}$ respectively). The inputs of this model are the delayed arterial gas concentrations after the lung gas exchange model $\left(\tilde{C}_{a \mathrm{O}}, \tilde{C}_{a \mathrm{CO} 2}\right)$ and the blood flows $\left(Q_{\text {out }, \text { sa }, e}\right)$ and volumes $\left(V_{\text {sves }}\right)$ of the systemic peripheral vessels of the CVS. The outputs (Figure 5) are the gas concentrations in the mixed venous blood $\left(C_{\text {svesO } 2}\right.$,

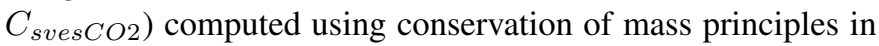
the venous compartments.

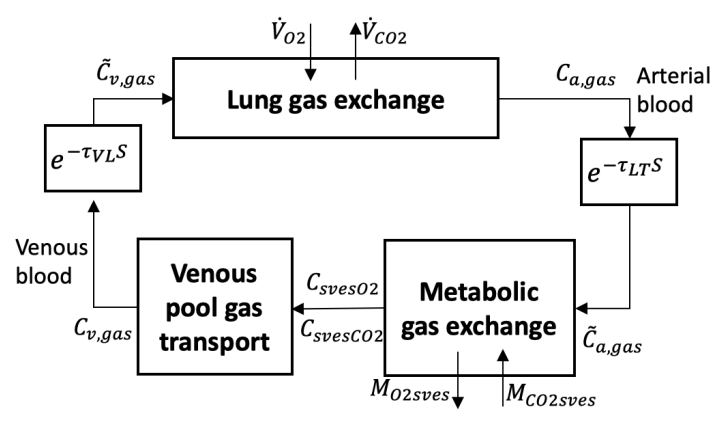

Fig. 5. Gas transport model. C, concentration; M, metabolic rate; $\mathrm{V}$, volume; a, arterial; $\mathrm{v}$, venous; $\tau_{L T}$ and $\tau_{V L}$, pure delays; sves, systemic peripheral vessels.

4) Gas transport: This sub-model represents the convey of $\mathrm{O}_{2}$ and $\mathrm{CO}_{2}$ throughout the cardiovascular system as is presented in Figure 5. The time that blood takes to transport the gases from the pulmonary capillaries to the systemic peripheral vessels and from the extra-thoracic veins to the pulmonary capillaries are defined by pure delays $\tau_{L T}$ and $\tau_{V L}$ respectively. Furthermore, $\tau_{L T}$ integrates the time that takes for the blood to travel from the lung to the pulse oximeter sensor site [22]. The transport throughout the intrathoracic and extra-thoracic veins is explicitly modelled applying conservation of mass principles to each systemic vein compartment.

\section{5) Neural control:}

a) Chemoreflex model: Peripheral and central chemoreflex sub-models (Figure 6), adapted from [8], were integrated into the model in order to represent modulations of breathing rhythm (BR) and the respiratory muscle maximal amplitude $\left(P_{\text {max }}\right)$ in response to $\mathrm{PaO}_{2}$ and $\mathrm{PaCO}_{2}$ modifications. The central chemoreceptors are assumed to be sensitive to $\mathrm{PaCO}_{2}$ and are represented as a combination of a pure delay $\left(D_{c}\right)$ and first-order systems, characterized by specific gains $\left(G_{c, A}\right.$, $\left.G_{c, f}\right)$ and time constants $\left(\tau_{c, f}, \tau_{c, A}\right)$. The peripheral chemoreceptors are assumed to be sensitive to $\mathrm{PaCO}_{2}$ and $\mathrm{PaO}_{2}$, and are described as a two-stage transduction mechanism. First, $\mathrm{PaO}_{2}$ and $\mathrm{PaCO}_{2}$ are transduced into the afferent electrical activity of the peripheral chemoreceptors $\left(f_{a c p}\right)$. Then, the second stage is represented as a pure delay $\left(D_{p}\right)$ and a set of first-order systems, defined by gains $\left(G_{p, A}, G_{p, f}\right)$ and time constants $\left(\tau_{p, f}, \tau_{p, A}\right)$. Contributions of each branch are summed to the baseline response of each regulated variable in order to define the maximum pressure of the respiratory muscles $\left(P_{\max }\right)$ and the breathing rythym (BR).

b) Baroreflex and pulmonary stretch receptors: Baroreflex model (Figure 7) is based on previous work of our laboratory [11], [23]. The afferent pathways, which regroup the afferent nerves connecting the baroreceptors to the pontomedullary cardiovascular control centers, are represented by a first-order transfer function (gain $K_{b}$ and time constant $T_{b}$ ). Efferent sympathetic and vagal pathways are represented by a combination of sigmoid functions, delays ( $D_{S}$ and $D_{V}$ respectively) and first-order filters, characterized by gains $\left(K_{S}, K_{V}\right)$ and a time constants $\left(T_{S}, T_{V}\right)$. Chronotropic control is performed through the sympathetic and parasympathetic branches, whereas systemic resistance, venous volume and ventricular elastance are only influenced by the sympathetic system. The pulmonary stretch receptors modulate the vagal branch of the baroreflex in relation to the changes of lung volume $V_{A}$ [24]. This is one of the main generators of the respiratory sinus arrhythmia [25].

\section{B. Clinical data}

The clinical database was obtained within the framework of the HYPNOS study, which was a multicenter, proof-ofconcept, open-label, prospective study, conducted at 5 hospitalbased university centers in France (Grenoble, Montpellier, Angers, Tours and Rennes). The study was approved by an independent Ethics Committee (Comit de Protection des Personnes, Grenoble, France, IRB 2014-A00339-38) and registered at ClincalTrials.gov with the identifier NCT03300037 (date of registration: 03/10/2017).

Subjects over 18 years, with severe obstructive sleep apnea (OSA), defined as an apnea-hypopnea index (AHI) > $30 / \mathrm{h}$ and less than $20 \%$ of central events, were eligible. All patients provided informed consent. Data from 24 patients were reported. The study population is typical of patients with severe OSA, predominantly middle-age, male, obese with frequent co-morbidities. All patients underwent a full standard polysomnography (PSG) study at an in-hospital sleep laboratory. The PSG study consisted in the acquisition, during a whole night, of a set of physiological signals (EEG, EMG, $\mathrm{ECG}, \mathrm{SaO}_{2}$, ventilation,...) that were analyzed after the acquisition in order to quantify the sleep quality of patients and characterize respiratory events [26]. The PSG system used was a Deltamed BrainBox 1042 (Natus, France) with the Cohrence software version 6.1.3.405, a Nonin Xpod Oximeter and a Braebon Ultima Airflow Pressure sensor. 


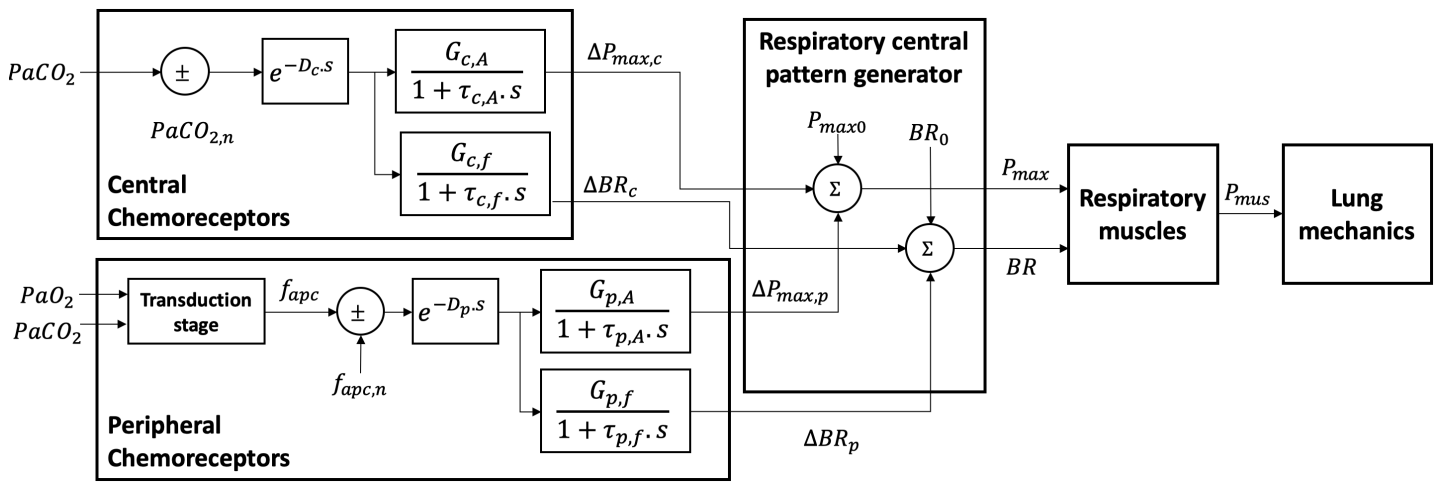

Fig. 6. Chemoreflex model. Pa, arterial partial pressure; $\boldsymbol{D}$, delay; BR, breathing rhythm; $\boldsymbol{P}_{\boldsymbol{m} u \boldsymbol{s}}$, activity of respiratory muscles; $\boldsymbol{P}_{\boldsymbol{m a x}}$, maximum amplitude of the activity of respiratory muscles; $\boldsymbol{f}_{a p c}$, afferent electrical activity of the peripheral chemoreceptors; $c$, central chemoreflex; $p$, peripheral chemoreflex; A, amplitude; f, frequency.

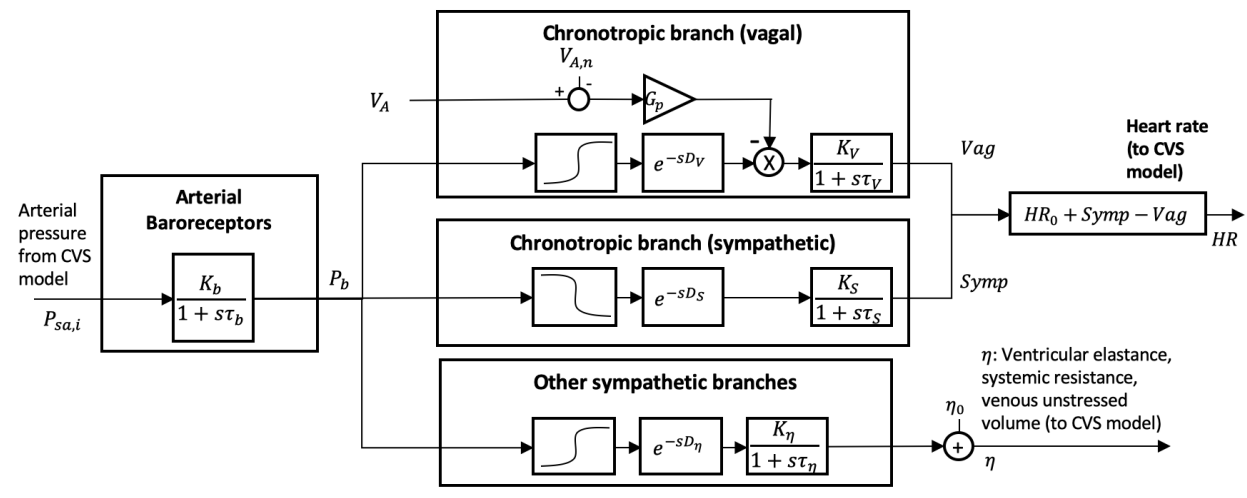

Fig. 7. Diagram of the baroreflex system. From the arterial pressure registered at the intrathoracic systemic circulation, the baroreflex system regulates the heart rate, the systemic resistance, the venous unstressed volume and the ventricular elastance. $\boldsymbol{K}$, gain; $\boldsymbol{D}$, delay; $\boldsymbol{\tau}$, time constant; $\boldsymbol{G}$, gain; $\boldsymbol{V}_{\boldsymbol{A}}$, lung volume.

PSG data acquired during the HYPNOS study were evaluated by a single scorer at the core-laboratory of the project (CHU Grenoble), with quality assured by an intra-scorer quality control process [27]. Annotations included the instant of occurrence of obstructive, central and mixed apneas, hypopneas, the sleep state and body position, among others.

1) Apnea selection for patient-specific identification: A subset of the annotated apnea events was selected for the patientspecific parameter identification of the model. The selection criteria was defined in accordance with the clinicians involved in the study, in order to increase the chances of observing pure acute cardio-respiratory effects and to minimize the mixed cardio-respiratory effects that are due to overlapping respiratory episodes. The criteria consist of:

1) obstructive apneas,

2) a minimum apnea duration of 20 seconds, in order to increase the chances of observing acute cardio-respiratory effects,

3) a minimum separation of 20 seconds between the apneic event of interest and the previous or consecutive event, in order to minimize the cardio-respiratory effects due to the other respiratory episodes.

2) Signal processing analysis: The RR interval time series of each patient was extracted from the ECG using a QRS complex detector developed by our team [28], in order to observe heart rate dynamics during the apneic event. A moving average filter was applied to the raw $\mathrm{SaO}_{2}$ signal, using a 5 second window. This filtered $\mathrm{SaO}_{2}$ is used in the cost function of the patient-specific identification.

\section{Patient-specific responses identification}

Obstructive apneas were simulated in our model by increasing the resistance of the upper airways $R_{u}$ of the respiratory model to $100000 \mathrm{~cm} \mathrm{H}_{2} \mathrm{O} \cdot \mathrm{s} \cdot \mathrm{l}^{-1}$ during the duration of the experimental apnea event, which was determined from the annotations of each patient's data. Baseline heart-rate $\left(H R_{0}\right)$ was fixed to produce the mean heart rate observed from the patient during the apnea event.

Results from a sensitivity analysis, previously published by our team [29], were used to select the 8 most significant model parameters to be identified :

- metabolic rates ( $M_{O 2 S v e s}$ and $\left.M_{C O 2 S v e s}\right)$,

- gains $\left(G_{c, A}, G_{p, A}\right)$ and time constants $\left(\tau_{c, A}, \tau_{p, A}\right)$ of the central and peripheral chemoreflex for the control of the amplitude of the activity of respiratory muscles,

- unstressed volume of the alveoli $\left(V_{u A}\right)$,

- gas transport delay between the lung and the systemic tissues $\tau_{L T}$. This parameter captures the lung to finger 
$\mathrm{SaO}_{2}$ delay.

The lower and upper bounds used in the identification process for each parameter were fixed as a function of values available in the literature: $80 \%$ for $V_{u A}, \tau_{L T}, M_{O 2 S v e s}$ and $M_{C O 2 \text { Sves }},+300 \% /-90 \%$ for $G_{c, A}$ and $G_{p, A}, 30 \%$ for $\tau_{c, A}$ and $\tau_{p, A}$.

1) Objective function: The objective function $g_{\epsilon}$ was defined similarly than previous works of our team [10], [24] with the goal of minimizing the error between the simulated and the experimental $\mathrm{SaO}_{2}$ in a time support going from 10 seconds before the beginning of the apnea to 60 seconds after the end of the apnea. The function to be minimized is:

$$
\begin{aligned}
& g_{\epsilon}=\omega_{1} *\left(\epsilon_{d}+\epsilon_{r}\right)+\omega_{2} * \epsilon_{M i n}+\omega_{3} * \epsilon_{\text {MinLoc }} \text {, with } \\
& \epsilon_{d}=\frac{1}{T_{\min }} \sum_{t_{e}=0}^{T_{\min }}\left|S a O 2^{\text {sim }}\left(t_{e}\right)-S a O 2_{f}^{e x p}\left(t_{e}\right)\right| \\
& \epsilon_{r}=\frac{1}{T_{e n d}-T_{\min }} \sum_{t_{e}=T_{m i n}}^{T_{\text {end }}}\left|S a O 2^{s i m}\left(t_{e}\right)-S a O 2_{f}^{\exp }\left(t_{e}\right)\right| \\
& \epsilon_{M i n}=\left|\min \left(\operatorname{SaO} 2^{\operatorname{sim}}\right)-\min \left(\operatorname{SaO} 2_{f}^{\exp }\right)\right| \\
& \epsilon_{\text {MinLoc }}=\left|S a O 2^{s i m}\left(T_{m i n}\right)-S a O 2_{f}^{\exp }\left(T_{\min }\right)\right|
\end{aligned}
$$

where $t_{e}$ corresponds to the time elapsed since the onset of the identification period, $\mathrm{SaO} 2^{\text {sim }}$ and $\mathrm{SaO}_{f}^{\exp }$ are, respectively, the simulated and experimental $\mathrm{SaO}_{2}, T_{\text {min }}$ is the time associated with the minimum value of $S a O 2_{f}^{e x p}$ and $T_{\text {end }}$ is the end of the identification period. $\omega_{i}$ corresponds to the weight given to each component of the objective function, with $\omega_{1}=0.7 ; \omega_{2}=0.4 ; \omega_{3}=0.4$ giving more importance to $\epsilon_{d}$ and $\epsilon_{r}$. A Differential Evolution (DE) algorithm was applied to find the optimal parameter set $P_{\text {opt }}$. DE is a population based, heuristic optimizer developed by [30] and it was implemented using the massively parallel framework PAGMO [31].

2) Error estimation between identified and experimental data: The difference between experimental and simulated signals were quantified using the root-mean-square error (RMSE) and the relative root-mean-square error (rRMSE):

$$
\begin{aligned}
R M S E & =\sqrt{\frac{1}{N} \sum_{t=0}^{N}\left(S a O 2^{\operatorname{sim}}(t)-S a O 2_{f}^{e x p}(t)\right)^{2}} \\
r R M S E & =\sqrt{\frac{1}{N} \sum_{t=0}^{N}\left(\frac{S a O 2^{\operatorname{sim}}(t)-S a O 2_{f}^{\exp }(k)}{S a O 2_{f}^{e x p}(t)}\right)^{2}}
\end{aligned}
$$

where $t$ corresponds to the current sample of the time series, $\mathrm{SaO} 2^{\text {sim }}$ and $\mathrm{SaO} 2_{f}^{\text {exp }}$ are, respectively, the simulated and experimental time series, $N$ is the number of samples of the time series.
TABLE I

LIST OF PATIENTS AND SELECTED APNEAS FOR PATIENT-SPECIFIC IDENTIFICATION.

\begin{tabular}{llll}
\hline Patients & Selected apneas & $\Delta \mathbf{S a O}_{\mathbf{2}}(\boldsymbol{\%})$ & BMI \\
\hline 2 & 32 & 5.26 & 24.91 \\
\hline 3 & 1 & 2.40 & 24.62 \\
\hline 5 & 10 & 7.33 & 27.41 \\
\hline 6 & 2 & 5.65 & 35.88 \\
\hline 8 & 2 & 3.51 & 25.55 \\
\hline 11 & 29 & 6.68 & 25.3 \\
\hline 12 & 7 & 9.42 & 26.82 \\
\hline 15 & 14 & 8.56 & 35.91 \\
\hline 16 & 6 & 5.26 & 22.59 \\
\hline 17 & 4 & 2.82 & 22.05 \\
\hline 10 patients & 107 apneas & &
\end{tabular}

3) Clustering: In order to identify clusters of phenotypically-similar responses to apnea, the set of all identified parameters was clustered by applying the K-means method [32]. The optimal number of clusters $k$ was obtained using the silhouette method defined by [33]. The objective here is to analyse similarities within the identified sets of parameters and to associate each cluster with a specific dynamic to obstructive events.

In order to visualize the results of the clustering, a principal component analysis (PCA) was performed in the model parameter space for unsupervised dimension reduction. The two principal components were used to visualize the first factorial plane, with a color assigned per cluster.

\section{REsults}

107 obstructive apneas distributed among 10 patients were selected from the HYPNOS dataset for patient-specific identification, according to the criteria presented in section IIB.1. Table I presents the number of obstructive events, the mean desaturation $\left(\triangle \mathrm{SaO}_{2}\right)$ during the selected obstructive episodes and the body mass index (BMI) for each selected patient. Within a range of 60 seconds after the end of the selected obstructive events, $66 \%$ of the selected apneas were followed by another apnea, $21 \%$ by a hypopnea and $13 \%$ had no consecutive respiratory event.

\section{A. Parameter identification}

Parameter values were identified for the selected 107 obstructive apnea events. Table II presents the median and interquartile range of RMSE and rRMSE per patient and for the whole database. Global median RMSE and rRMSE are respectively equal to $1.3892 \%$ and 0.0154 . The simulated $\mathrm{SaO}_{2}$ reproduces closely the experimental $\mathrm{SaO}_{2}$ in all the model identifications.

\section{B. $\mathrm{SaO}_{2}$ dynamics reproduced by the identified model}

The model was able to reproduce different dynamics observed in the experimental obstructive apneas. Two examples of representative patients are described in this section:

1) Example 1: obstructive apnea with normal recovery. Figure 8-A shows a typical response to an obstructive event, with a significant decrease of the $\mathrm{SaO}_{2}$ during 
TABLE II

MEDIAN AND INTERQUARTILE RANGE OF THE RMSE AND RRMSE BETWEEN THE IDENTIFIED MODEL SIMULATION OF SAO 2 AND THE EXPERIMENTAL $\mathrm{SAO}_{2}$ PER PATIENT AND FOR THE TOTAL DATABASE.

\begin{tabular}{llllll} 
Patient & Apneas & $\begin{array}{l}\text { Median } \\
\text { RMSE (\%) }\end{array}$ & $\begin{array}{l}\text { Iqr } \\
\text { RMSE }(\%)\end{array}$ & $\begin{array}{l}\text { Median } \\
\text { rRMSE }\end{array}$ & $\begin{array}{l}\text { Iqr } \\
\text { rRMSE }\end{array}$ \\
\hline 2 & 32 & 1.200 & 0.484 & 0.013 & 0.005 \\
\hline 3 & 1 & 1.199 & 0.000 & 0.013 & 0.000 \\
\hline 5 & 10 & 1.273 & 0.510 & 0.014 & 0.005 \\
\hline 6 & 2 & 2.067 & 0.939 & 0.022 & 0.011 \\
\hline 8 & 2 & 1.921 & 1.064 & 0.021 & 0.012 \\
\hline 11 & 29 & 1.798 & 1.010 & 0.019 & 0.011 \\
\hline 12 & 7 & 1.384 & 0.312 & 0.016 & 0.004 \\
\hline 15 & 14 & 1.972 & 0.562 & 0.021 & 0.006 \\
\hline 16 & 6 & 1.336 & 0.484 & 0.015 & 0.006 \\
\hline 17 & 4 & 0.768 & 0.293 & 0.008 & 0.003 \\
Total & 107 & 1.389 & 0.656 & 0.015 & 0.008
\end{tabular}

apnea and an increase of ventilation after the event [34]. RMSE and rRMSE between experimental and simulated $\mathrm{SaO}_{2}$ are respectively $1.7302 \%$ and 0.0186 .

2) Example 2: obstructive apnea followed by another apnea. Figure 8-B shows an apnea event followed by a fast increase in ventilation and a subsequent apnea. RMSE and rRMSE are $1.17 \%$ and 0.0127 .

Although the experimental nasal pressure was not included in the objective function of the identification algorithm, the simulated lung volume was able to reproduce correctly the observed respiratory activity, in particular after the apnea event, in both cases.

\section{Characterization of the desaturation response to obstructive apneas}

The optimal number of clusters $k$ obtained using the silhouette method was $k=2$ (Figure 9). K-means clustering method was applied to the 107 sets of parameters, and barycenters of the two obtained clusters were extracted. Barycenter 1 is characterized by high gain values and short time constants for the chemoreflex, long lung-tissue gas transport time $\left(\tau_{L T}\right)$, low metabolic rates and low tidal volume. Parameters of Barycenter 2 are close to the reference parameters used by the model for a normal adult. Taking advantage of the interpretability provided by the model, two simulations were performed, using as parameter values those obtained from each barycenter (Figure 10). This is useful to better understand the physiology behind each cluster response to obstructive events and thus to better characterize them.

In cluster 1 , the simulated obstructive event generates a consecutive central apnea followed by ventilation instability with a crescendo-diminuendo respiratory pattern. Previous to the apnea, the respiratory tidal volume $\left(\mathrm{V}_{\mathrm{T}}\right)$ is almost half of the normal value $\left(0.5 \mathrm{~L}\right.$ [35]) resulting in a low $\mathrm{PaO}_{2}$. During the obstructive apnea, the $\mathrm{PaO}_{2}$ and the $\mathrm{SaO}_{2}$ show a significant drop and the $\mathrm{PaCO}_{2}$ is slightly increased. Due to the higher gain and the short time constants of both the central and peripheral chemoreceptors, there is an overcompensation of $\mathrm{PaO}_{2}$ and $\mathrm{PaCO}_{2}$, and the respiratory amplitude oscillates.

Cluster 2 shows a typical response to obstructive apnea followed by recovery of spontaneous breathing. Before the event, the lung volume is normal. During the obstruction, $\mathrm{SaO}_{2}$ and $\mathrm{PaO}_{2}$ decrease and the $\mathrm{PaCO}_{2}$ increases. After the apnea event, there is an increase of ventilatory effort and the respiration stabilizes.

\section{DISCUSSION}

To our knowledge, the proposed model is the first integrated representation of cardio-respiratory interactions, for the analysis of acute responses to obstructive apneas. The main contributions of this work are: a) the integration of physiological functions involved in complex interactions between the respiratory, neural and cardiac systems, b) the patient-specific and event-specific analysis of cardio-respiratory interactions during adult sleep apnea, through model parameter identification, c) the characterization of the desaturation response to obstructive apneas.

\section{A. Integrated cardio-respiratory model of adult physiology}

The structural and functional integration of physiological functions, involved in the complex interactions between the respiratory, cardiac and nervous systems, was performed through a multi-resolution modeling approach. This approach is compatible with a systems physiology representation at the scale of the organism, while permitting the coupling of higher-resolution models to account for detailed local effects [36]. Formally heterogeneous models were coupled in order to obtain an original representation of: i) mechanisms involved in OSA, ii) the interactions between discrete cardiac electrical conduction system and lumped-parameter cardio-respiratory models, iii) integration of metabolism in circulatory model and iv) an analytic representation of the respiration pattern adapted to adults. This approach appears to be particularly adapted to the investigation of the clinical applications related to apnea. The complete model constitutes a typical example of integrative modeling in physiology, and adult digital patient [37]. The acute dynamics related to obstructive apneas are represented while maintaining a reduced number of parameters (151 parameters) compared to other integrated models in the literature [6], [7], [8] (with 472, 557 and 240 parameters respectively), making it more suitable to patient-specific and event-specific identifications.

\section{B. Patient-specific and event-specific analysis of desaturation dynamic on a PSG database.}

This work constitutes the first successful patient-specific and event-specific parameter identification of the desaturation response to obstructive apneas on a polysomnography database using an integrated adult model.

The desaturation dynamics and, for some events, the nasal pressure were reproduced in 107 obstructive apneas observed from 10 patients, by using as input the duration of the apnea and the observed $\mathrm{SaO}_{2}$ signal.

In several cases, the following respiratory events (apnea, hypopnea, normal recovery) were also reproduced. This result is explained by the definition of the objective function, that 

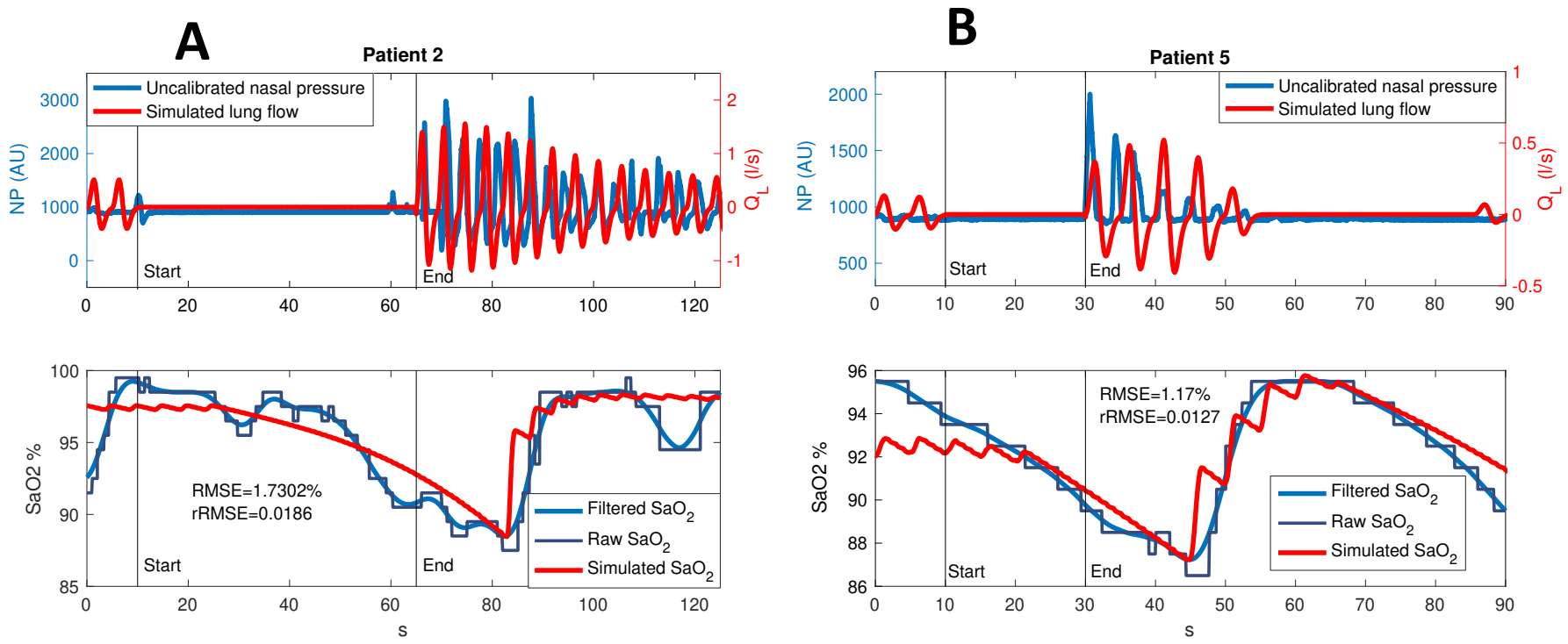

Fig. 8. Example of two dynamics simulated by the model, after patient- and event-specific parameter identification. A) obstructive apnea with normal recovery B) obstructive apnea followed by another apnea. Comparison between apnea events of PASITHEA database and simulation with identified parameters using the adult model. Top to bottom: Experimental uncalibrated nasal pressure (Blue) and simulated lung flow (Red); Filtered experimental $\mathrm{SaO}_{2}$ (Blue), raw experimental $\mathrm{SaO}_{2}$ (Dark blue) and simulated $\mathrm{SaO}_{2}(R e d)$.

- High loop gain.

- Low tidal volume and $\mathrm{PaO}_{2}$.

- Low $\mathrm{CO}_{2}$ production.

- Long circulation transport.

- Ventilatory instability.

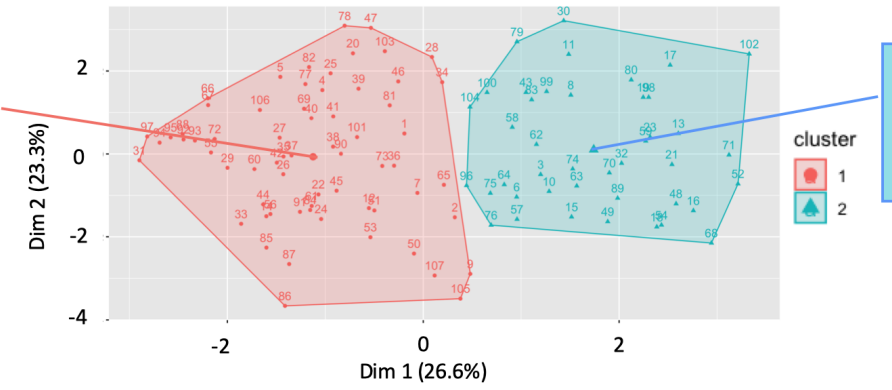

- Regular behavior.

- Normal apnea dynamic with normal recovery.

Fig. 9. K-mean clustering on the 107 set of parameters identified for the adult cardio-respiratory model using the PASITHEA database. Each dot in this figure corresponds to a set of parameters identified for a given apnea episode of a given patient. Two clusters were found: 1 (red), 2 (blue).
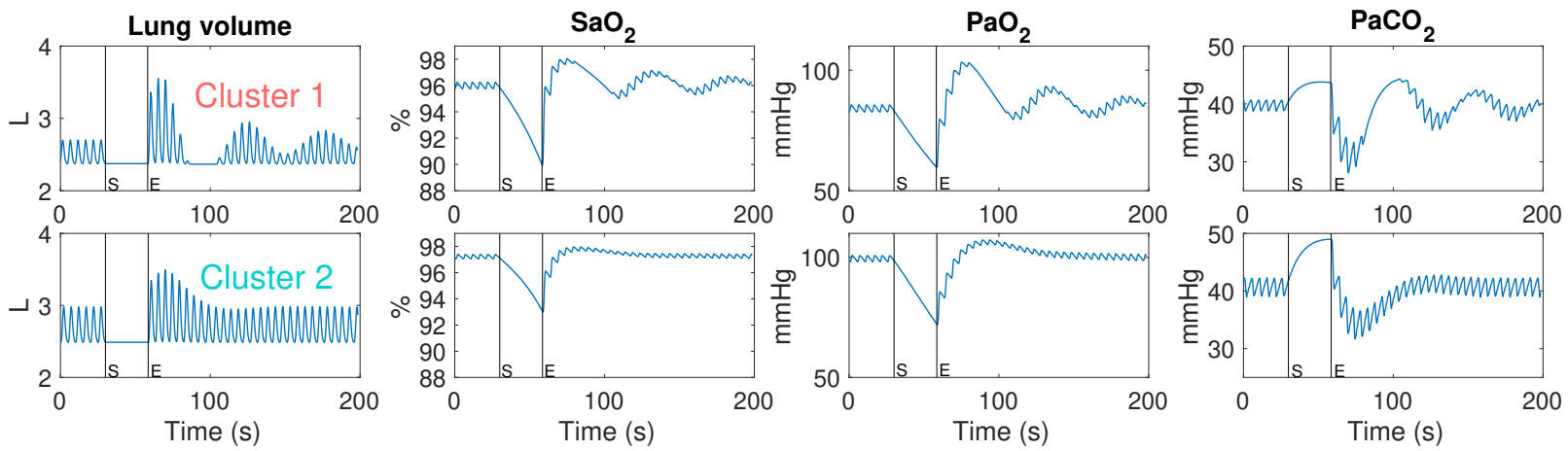

Fig. 10. Simulations of an obstructive event using the adult cardio-respiratory model with the parameters of the barycenters of the two cluster found with the K-means clustering method. Each row presents the simulation of the lung volume, the $\mathrm{SaO}_{2}$, the arterial partial pressure of oxygen $\left(\mathrm{PaO}_{2}\right)$, the arterial partial pressure of $\mathrm{CO}_{2}$ and the heart rate per each of two clusters.

includes a 60 -second window after the end of the obstructive apnea, taking thus into consideration the desaturation produced by consecutive events. The shape of $\mathrm{SaO}_{2}$, during and after an apnea event, can provide useful information to better characterize the ventilatory dynamics of a patient.
Parameter identification of integrated cardio-respiratory models is usually considered as challenging task [8]. Previous sensitivity analysis provided valuable information about the most influential parameters on $\mathrm{SaO}_{2}$, which could be selected for the identification (8 parameters out of 151), allowing for 
a significant reduction of the parameter space, thus improving identifiability.

\section{Characterization of the desaturation response to obstructive apneas}

After parameter identification, a clustering method was applied to the resulting model parameter space. Compared to the common approach of applying clustering to the observation space, the interpretability is here increased because the cluster interpretation is based on physiological parameters, rather than in observations or features, allowing for a more meaningful phenotyping. The proposed clustering-based characterization of the desaturation response to obstructive apneas resulted in two different clusters with different physiological responses to apnea and a phenotype was thus assigned to each of these clusters.

Clusters 1 is characterized by ventilatory instability. The disturbance produced by the obstructive event produces a periodic breathing (PB) / Cheyne-Stokes respiration (CSR). The chemoreflex of this cluster is characterized by high gains and short time constants, having as a direct consequence a high controller loop gain and chemoreflex sensitivity. These two aspects are highly relevant for PB and ventilation instability [38], [39]. Moreover, cluster 1 presents long circulation delays, usually related to PB [40]. The dynamics of cluster 1 are associated to low $\mathrm{PaO}_{2}$ resembling Cheyne-Stokes respiration due to hypoxaemia in congestive heart failure through peripheral chemoreceptor stimulation [41]. The low tidal volume shown by this cluster contributes to this hypoxemia during apnea [42]. Moreover, the disturbance produced by the obstructive apnea caused hyperventilation that dropped the value of $\mathrm{PaCO}_{2}$ significantly. These dynamics are the commonly associated with of all forms of $\mathrm{PB}$, where $\mathrm{PaCO}_{2}$ drops below the apnoeic threshold, triggering a central apnea [43]. Similarly, increased central hypercapnic ventilatory responsiveness has been reported to occur in Cheyne-Stokes respiration [44]. Furthermore, the metabolic rates in cluster 1 are low, affecting the change of $\mathrm{PaO}_{2}$ and $\mathrm{PaCO}_{2}$ during the obstructive event, where the metabolism plays the main role [45]. Metabolic rates are directly related to the plant gain of the ventilatory system, that is an important factor for sleep apnea [46].

Cluster 2 is associated with a typical response to obstructive apnea, with moderate chemoreflex gains and metabolic rates [34].

These phenotypes may be used as an instrument for the identification of patients with higher risk of ventilation instability and periodic respiration in order to improve and personalize treatment. Such a modelling might be useful for predicting response to CPAP and needs to shift to alternative ventilatory support, including adapted servo-ventilation [47], [48].

\section{Limitations}

Events like sighs and micro-arousals are not represented within the model, and they can have an impact on the dynamics of sleep apneas. Sighs are related to posterior apneas, hypoventilation, or slowing the respiratory frequency, and they can produce up to $2 \%$ change of the $\mathrm{SaO}_{2}$ [49]. The autonomic responses linked to sleep arousals are a crucial component of the resumption of ventilation [50], [51]. Similarly, the chemoreflex input to sympathetic activity [52], [53] is not included in the model. Moreover, the simulation of obstruction during apnea events is simplified with a step increase in the upper airway resistance, although more complex dynamics are observed in real obstructive events. The model does not integrate the capabilities to produce consecutive obstructive or mixed apneas, hence secondary events can only be central in nature, as a consequence of high loop gain. The observed "high loop gain" could reflect overall system periodicity via a range of mechanisms (e.g. obstruction following sleep onset or obstruction mediated by low chemoreflex drive to both pump and dilator muscles). Loop gain estimation [44] should be included in future clinical protocols for further validation.

The objective function used for the identification was mainly based on the shape of $\mathrm{SaO}_{2}$. In several cases, the identified model was able to reproduce the experimental $\mathrm{SaO}_{2}$ and the uncalibrated nasal pressure. But in other cases, the simulated $\mathrm{SaO}_{2}$ resembles the experimental $\mathrm{SaO}_{2}$, but the respiration is different. In future works, the nasal pressure signal will be taken into account in the objective function. Furthermore, only apneas with a duration of more than 20 seconds were used in the patient-specific identification for a more pronounce cardiorespiratory effect, excluding shorter events that will be studied in further work.

\section{CONCLUSION}

The cardio-respiratory model was able to reproduce the experimental $\mathrm{SaO}_{2}$ during and after an OSA event in a database of 107 obstructive apneas observed from 10 patients. After the identification procedure, two clusters were found by applying the K-means method to the model parameter space. These clusters describe different dynamics related to sleep apnea and periodic breathing. The first cluster was related to ventilation instability due to high loop gain and periodic/Cheynes-Stokes breathing. The second cluster represented the typical response to an obstructive event. The predominance of any of these clusters in a patient offers information about the dynamic of the patient to obstructive apneas and it can help in the decisionmaking between different treatments like CPAP or oxygen therapy. In future works, the model-based methodology will be applied to optimize personalized therapy delivery, by coupling to our model virtual or physical therapeutic devices, as proposed in our previous works [11]. Mixed cardio-respiratory effects, due to overlapping respiratory episodes, and heart rate variations will also be analyzed.

\section{REFERENCES}

[1] P. Lévy, M. Kohler, W. T. McNicholas, F. Barbé, R. D. McEvoy, V. K. Somers, L. Lavie, and J. L. Pépin, "Obstructive sleep apnoea syndrome," Nature Reviews Disease Primers, vol. 1, 62015.

[2] A. V. Benjafield, N. T. Ayas, P. R. Eastwood, R. Heinzer, M. S. Ip, M. J. Morrell, C. M. Nunez, S. R. Patel, T. Penzel, J. L. D. Pépin, P. E. Peppard, S. Sinha, S. Tufik, K. Valentine, and A. Malhotra, "Estimation of the global prevalence and burden of obstructive sleep apnoea: a literature-based analysis," The Lancet Respiratory Medicine, vol. 7, pp. 687-698, 82019. 
[3] C. D. Sá Couto, W. L. van Meurs, J. A. Goodwin, and P. Andriessen, "A Model for Educational Simulation of Neonatal Cardiovascular Pathophysiology," Simulation in Healthcare: The Journal of the Society for Simulation in Healthcare, vol. 1, no. Inaugural, pp. 4-9, 2006.

[4] L. M. Ellwein, S. R. Pope, A. Xie, J. Batzel, C. T. Kelley, and M. S. Olufsen, "Modeling cardiovascular and respiratory dynamics in congestive heart failure," Mathematical Biosciences, vol. 241, pp. 56-74, 2013.

[5] V. Le Rolle, N. Samson, J.-p. Praud, A. I. Hernández, V. L. Rolle, N. Samson, J.-p. Praud, and A. I. Hernández, "Mathematical modeling of respiratory system mechanics in the newborn lamb. To cite this version : HAL Id : hal-00880028," 2013.

[6] L. Cheng, O. Ivanova, H. H. Fan, and M. C. Khoo, "An integrative model of respiratory and cardiovascular control in sleep-disordered breathing," Respiratory Physiology and Neurobiology, vol. 174, no. 1-2, pp. 4-28, 2010.

[7] L. Cheng and M. C. Khoo, "Modeling the autonomic and metabolic effects of obstructive sleep apnea: A simulation study," Frontiers in Physiology, vol. 2 JAN, 2012.

[8] A. Albanese, L. Cheng, M. Ursino, and N. W. Chbat, "An integrated mathematical model of the human cardiopulmonary system: Model development," American Journal of Physiology - Heart and Circulatory Physiology, p. ajpheart.00230.2014, 2015.

[9] M. Calvo, V. Le Rolle, D. Romero, N. Béhar, P. Gomis, P. Mabo, and A. I. Hernández, "Model-based analysis of the autonomic response to head-up tilt testing in Brugada syndrome," Computers in Biology and Medicine, vol. 103, pp. 82-92, 122018.

[10] M. Calvo, V. Le Rolle, D. Romero, N. Béhar, P. Gomis, P. Mabo, and A. I. Hernández, "Recursive model identification for the analysis of the autonomic response to exercise testing in Brugada syndrome," Artificial Intelligence in Medicine, vol. 97, pp. 98-104, 62019.

[11] H. M. Romero Ugalde, D. Ojeda, V. Le Rolle, D. Andreu, D. Guiraud, J. L. Bonnet, C. Henry, N. Karam, A. Hagege, P. Mabo, G. Carrault, and A. I. Hernandez, "Model-Based Design and Experimental Validation of Control Modules for Neuromodulation Devices," IEEE Transactions on Biomedical Engineering, vol. 63, no. 7, pp. 1551-1558, 2016.

[12] V. Le Rolle, A. I. Hernández, P.-Y. Richard, E. Donal, and G. Carrault, "Model-based analysis of myocardial strain data acquired by tissue Doppler imaging," Artificial Intelligence in Medicine, vol. 44, pp. 201219, 112008

[13] S. Al-Omar, V. Le Rolle, P. Pladys, N. Samson, A. Hernandez, G. Carrault, and J. P. Praud, "Influence of nasal CPAP on cardiorespiratory control in healthy neonate," Journal of Applied Physiology, vol. 127, no. 5, pp. 1370-1385, 2019.

[14] G. Guerrero, V. Le Rolle, J. L. Pépin, and A. Hernández, "Parameter identification of a cardio-respiratory model for the study of obstructive sleep apnea.," in VPH2020, (Paris), 2020.

[15] A. I. Hernandez, V. Le Rolle, A. Defontaine, and G. Carrault, "A multiformalism and multiresolution modelling environment: application to the cardiovascular system and its regulation," Philosophical Transactions of the Royal Society A: Mathematical, Physical and Engineering Sciences, vol. 367, pp. 4923-4940, 122009.

[16] A. I. Hernández, G. Carrault, F. Mora, and A. Bardou, "Model-based interpretation of cardiac beats by evolutionary algorithms: Signal and model interaction," Artificial Intelligence in Medicine, vol. 26, no. 3, pp. 211-235, 2002.

[17] D. Ojeda, V. Le Rolle, K. Tse Ve Koon, C. Thebault, E. Donal, A. I. Hernandez, K. Tse, V. Koon, C. Thebault, E. Donal, A. I. Hernandez, and A. I. Hernández, "Towards an Atrio-Ventricular Delay optimization assessed by a computer model for Cardiac Resynchronization Therapy," Proceedings of SPIE - The International Society for Optical Engineering, vol. 8922, pp. 333-336, 2013.

[18] N. Stergiopulos, J. J. Meister, and N. Westerhof, "Determinants of stroke volume and systolic and diastolic aortic pressure," American Journal of Physiology-Heart and Circulatory Physiology, vol. 270, pp. H2050H2059, 61996.

[19] K. Lu, J. W. Clark, F. H. Ghorbel, D. L. Ware, and a. Bidani, "A human cardiopulmonary system model applied to the analysis of the Valsalva maneuver.," American journal of physiology. Heart and circulatory physiology, vol. 281, no. 6, pp. H2661-H2679, 2001.

[20] G. Avanzolini, P. Barbini, F. Bernardi, G. Cevenini, and G. Gnudi, "Role of the mechanical properties of tracheobronchial airways in determining the respiratory resistance time course," Annals of Biomedical Engineering, vol. 29, no. 7, pp. 575-586, 2001.

[21] J. L. Spencer, E. Firouztale, and R. B. Mellins, "Computational expressions for blood oxygen and carbon dioxide concentrations," Annals of Biomedical Engineering, vol. 7, no. 1, pp. 59-66, 1979.
[22] C. F. Poets, "Apnea of prematurity: What can observational studies tell us about pathophysiology?," 82010.

[23] V. Le Rolle, A. I. Hernández, P. Y. Richard, and G. Carrault, "An autonomic nervous system model applied to the analysis of orthostatic tests," Modelling and Simulation in Engineering, vol. 2008, no. i, 2008.

[24] V. Le Rolle, A. Beuchee, J. P. Praud, N. Samson, P. Pladys, and A. I. Hernández, "Recursive identification of an arterial baroreflex model for the evaluation of cardiovascular autonomic modulation," Computers in Biology and Medicine, vol. 66, pp. 287-294, 2015.

[25] L. A. Rolak, Y. Harati, and S. Izadyar, "Autonomic Nervous System," Neurology Secrets, pp. 204-226, 12010

[26] J. L. Pépin, R. Tamisier, J. P. Baguet, B. Lepaulle, F. Arbib, N. Arnol, J. F. Timsit, and P. Lévy, "Fixed-pressure CPAP versus auto-adjusting CPAP: comparison of efficacy on blood pressure in obstructive sleep apnoea, a randomised clinical trial," Thorax, vol. 71, pp. 726-733, 8 2016.

[27] A. I. Hernández, D. Pérez, D. Feuerstein, C. Loiodice, L. Graindorge, G. Guerrero, N. Limousin, F. Gagnadoux, Y. Dauvilliers, R. Tamisier, A. Prigent, P. Mabo, A. Amblard, L. Senhadji, and J. L. Pépin, "Kinesthetic stimulation for obstructive sleep apnea syndrome: An "onoff" proof of concept trial," Scientific Reports, vol. 8, no. 1, pp. 1-7, 2018.

[28] M. Doyen, D. Ge, A. Beuchée, G. Carrault, and A. I. Hernández, "Robust, real-time generic detector based on a multi-feature probabilistic method," PLOS ONE, vol. 14, no. 10, 2019.

[29] G. Guerrero, V. Le Rolle, and A. I. Hernández, "Sensitivity Analysis of a Cardiorespiratory Model for the Study of Sleep Apnea," CINC, 2018.

[30] R. Storn and K. Price, "Differential Evolution A Simple and Efficient Heuristic for global Optimization over Continuous Spaces," Journal of Global Optimization, vol. 11, no. 4, pp. 341-359, 1997.

[31] F. Biscani and D. Izzo, "esa/pagmo2: pagmo 2.10," 12019.

[32] S. P. Lloyd, "Least Squares Quantization in PCM," IEEE Transactions on Information Theory, vol. 28, no. 2, pp. 129-137, 1982.

[33] P. J. Rousseeuw, "Silhouettes: A graphical aid to the interpretation and validation of cluster analysis," Journal of Computational and Applied Mathematics, vol. 20, pp. 53-65, nov 1987.

[34] M. G. Levitzky, "Using the pathophysiology of obstructive sleep apnea to teach cardiopulmonary integration," American Journal of Physiology - Advances in Physiology Education, vol. 32, no. 3, pp. 196-202, 2008.

[35] A. C. Guyton and J. E. Hall, Textbook of medical physiology. 2010.

[36] A. I. Hernández, V. Le Rolle, D. Ojeda, P. Baconnier, J. FontecaveJallon, F. Guillaud, T. Grosse, R. G. Moss, P. Hannaert, and S. R. Thomas, "Integration of detailed modules in a core model of body fluid homeostasis and blood pressure regulation," Progress in Biophysics and Molecular Biology, vol. 107, pp. 169-182, 102011.

[37] C. G. Rizo, "The Digital and In Silico Therapeutics Revolution," pp. 197-214, Springer, Cham, 2018.

[38] M. C. Khoo, "Determinants of ventilatory instability and variability," in Respiration Physiology, vol. 122, pp. 167-182, Elsevier, 92000.

[39] D. P. Francis, K. Willson, L. C. Davies, A. J. Coats, and M. Piepoli, "Quantitative general theory for periodic breathing in chronic heart failure and its clinical implications," Circulation, vol. 102, pp. 22142221, 102000.

[40] N. S. Cherniack and G. S. Longobardo, "Mathematical models of periodic breathing and their usefulness in understanding cardiovascular and respiratory disorders," Experimental Physiology, vol. 91, pp. 295305, 32006.

[41] M. T. Naughton, "Pathophysiology and treatment of Cheyne-Stokes respiration," 61998.

[42] L. J. Findley, A. L. Ries, G. M. Tisi, and P. D. Wagner, "Hypoxemia during apnea in normal subjects: Mechanisms and impact of lung volume," Journal of Applied Physiology Respiratory Environmental and Exercise Physiology, vol. 55, no. 6, pp. 1777-1783, 1983.

[43] P. Solin, T. Roebuck, D. P. Johns, E. H. Walters, and M. T. Naughton, "Peripheral and central ventilatory responses in central sleep apnea with and without congestive heart failure," American Journal of Respiratory and Critical Care Medicine, vol. 162, pp. 2194-2200, 122000.

[44] A. Wellman, D. J. Eckert, A. S. Jordan, B. A. Edwards, C. L. Passaglia, A. C. Jackson, S. Gautam, R. L. Owens, A. Malhotra, and D. P. White, "A method for measuring and modeling the physiological traits causing obstructive sleep apnea," Journal of Applied Physiology, vol. 110, pp. 1627-1637, 62011.

[45] S. A. Sands, B. A. Edwards, V. J. Kelly, M. R. Davidson, M. H. Wilkinson, and P. J. Berger, "A model analysis of arterial oxygen desaturation during apnea in preterm infants," PLoS Computational Biology, vol. 5, no. 12, 2009. 
[46] M. T. Naughton, "Loop gain in apnea gaining: Control or controlling the gain?," 12010.

[47] J. L. D. Pépin, H. Woehrle, D. Liu, S. Shao, J. P. Armitstead, P. A. Cistulli, A. V. Benjafield, and A. Malhotra, "Adherence to positive airway therapy after switching from CPAP to ASV: A big data analysis," Journal of Clinical Sleep Medicine, vol. 14, pp. 57-63, 12018.

[48] S. Baillieul, B. Revol, I. Jullian-Desayes, M. Joyeux-Faure, R. Tamisier, and J. L. Pépin, "Diagnosis and management of central sleep apnea syndrome," 62019.

[49] R. Perez Padilla, P. West, and M. H. Kryger, "Sighs during sleep in adult humans," Sleep, 1983.

[50] J. Spaak, Z. J. Egri, T. Kubo, E. Yu, S. I. Ando, Y. Kaneko, K. Usui, T. D. Bradley, and J. S. Floras, "Muscle sympathetic nerve activity during wakefulness in heart failure patients with and without sleep apnea," Hypertension, vol. 46, pp. 1327-1332, 122005.

[51] K. S. Taylor, H. Murai, P. J. Millar, N. Haruki, D. S. Kimmerly, B. L. Morris, G. Tomlinson, T. Douglas Bradley, and J. S. Floras, "Arousal From Sleep and Sympathetic Excitation During Wakefulness Obstructive Sleep Apnea," Hypertension, vol. 68, pp. 1467-1474, 2016.

[52] K. Narkiewicz, P. J. Van De Borne, N. Montano, M. E. Dyken, B. G. Phillips, and V. K. Somers, "Contribution of tonic chemoreflex activation to sympathetic activity and blood pressure in patients with obstructive sleep apnea," Circulation, vol. 97, pp. 943-945, mar 1998.

[53] N. J. Marcus, R. Del Rio, E. P. Schultz, X. H. Xia, and H. D. Schultz, "Carotid body denervation improves autonomic and cardiac function and attenuates disordered breathing in congestive heart failure," Journal of Physiology, vol. 592, pp. 391-408, jan 2014. 